\title{
RÉSINES ÉPOXY RÉTICULÉES PAR DES POLYAMINES: STRUCTURE ET PROPRIÉTÉS
}

\section{BARRÈRE et F. DAL MASO}

Institut français du pétrole ${ }^{l}$
(1) 1 et 4 , avenue de Bois-Préau 92582 Rueil-Malmaison Cedex - France
Cet article fait le point sur l'état actuel des connaissances concernant les résines époxy réticulées par des polyamines, et plus particulièrement sur :

- la réticulation;

- la structure et la morphologie des systèmes réticulés et les paramètres qui les influencent;

- les relations entre la structure et les propriétés sur une très large gamme de températures.

Malgré les nombreuses études réalisées, beaucoup de points apparaissent encore controversés, notamment en ce qui concerne les relations structure/propriétés dans le domaine des températures d'utilisation, c'est-à-dire entre la transition secondaire $\beta$ et la transition $\alpha$ (transition vitreuse). S'il est maintenant admis que le module à l'état vitreux est gouverné par l'importance des mouvements des séquences hydroxypropyléther formées lors de la réticulation, les résultats concernant les propriétés mécaniques aux grandes déformations (allongement et contrainte à rupture, énergie de propagation de fissures) sont beaucoup plus discutés. Les tendances obtenues semblent dépendre de la manière utilisée pour faire varier la densité de réticulation (cuisson, proportions du mélange, extenseurs de mailles, prépolymères de différentes longueurs, etc.), du système ou encore de l'auteur. Les désaccords entre chercheurs pourraient provenir des conditions de préparation des échantillons, notamment de la vitesse de refroidissement en fin de cuisson et des conditions dans lesquelles sont réalisés les essais mécaniques, comme la vitesse de déformation.

\section{EPOXY RESINS CROSSLINKED WITH POLYAMINES: STRUCTURE AND PROPERTIES}

This paper sums up the current state-of-the-art on epoxy resins crosslinked with polyamines, and more particularly on:

- the crosslinking reactions;

- the structure and morphology of crosslinked systems and the parameters that influence them;

- the relationships between structure and properties over a wide range of temperatures.

In spite of numerous studies devoted to these polymers, many points remain controversial, notably concerning the relationships between structure and properties in the temperature range of interest to most users, which is between the $\beta$ transition temperature and the $\alpha$ transition temperature (glass transition). If it is now widely accepted that the modulus in the glassy state is mainly controlled by the amplitude of the motions of hydroxypropylether groups created during the crosslinking reactions, the results regarding the ultimate mechanical properties (stress and deformation at break, crack propagation energy) are much more 
debated. The trends obtained appear to depend upon either the way the crosslink density has been modified (cure cycle, mixture composition, chain extenders, prepolymers of different lengths and flexibilities), or the polymers studied or even the authors. The discrepancies between authors could be due to the sample preparation, particularly the cooling rate at the end of the cure cycle, or the testing conditions, e.g. the strain rate.

\section{RESINAS EPOXÍDICAS RETICULADAS POR POLIAMINAS : ESTRUCTURA Y PROPIEDADES}

En el presente artículo se describe la situación actual de los conocimientos relativos a las resinas epoxídicas reticuladas por poliaminas $y$, en particular, acerca de :

- la reticulación;

- la estructura y la morfología de los sistemas reticulados y los parámetros que influyen sobre éstos ;

- las relaciones entre la estructura y las propiedades en una escala muy amplia de temperaturas.

A pesar de los numerosos estudios llevados a cabo, aún aparecen numerosos puntos sujetos a controversia, y fundamentalmente, por lo que respecta a las relaciones estructura/propiedades en el aspecto de las temperaturas de utilización, o sea, entre la transición secundaria $\beta$ y la transición $\alpha$ (transición vítrea). Si bien se admite actualmente que el módulo en estado vítreo depende de la importancia de los movimientos de las secuencias hidroxipropiléter que se forman durante la reticulación, los resultados relativos a las propiedades mecánicas de las grandes deformaciones (alargamiento y tensión de ruptura, energía de propagación de las fisuras) se han puesto en tela de juicio de forma mucho más aguda. Las tendencias obtenidas parecen depender del método utilizado para introducir variaciones en cuanto a la densidad de reticulación (cocción, proporciones de la mezcla, extensores de mallas, prepolímeros de diversas longitudes, etc.), así como del sistema e, incluso, del propio autor. Las divergencias entre investigadores pueden proceder de las condiciones de preparación de las muestras, y en particular, de la velocidad de enfriamiento en la fase final de cocción y de las condiciones en que se llevan a cabo los ensayos mecánicos, como ocurre con la velocidad de deformación.

\section{INTRODUCTION}

Parmi les matières plastiques courantes, les résines époxy ont une place à part du fait de leur polyvalence. Ainsi sont-elles employées par toutes les industries, en tant que matériau de structure (matrice de matériaux composites) ou de surface (revêtement, adhésif, etc.). L'industrie pétrolière ne fait pas exception et de nombreuses applications font appel aux propriétés particulières de ces résines, comme le faible poids, la résistance à la corrosion ou le caractère adhésif.

Une abondante littérature fait état des recherches entreprises sur ces résines. Quelques livres font office de documents de référence et réunissent une part importante des connaissances acquises [1] et [2]. Néanmoins, plusieurs résultats récents permettent d'aller plus loin dans la compréhension du comportement de ces polymères. En plus d'une synthèse de ces résultats, cet article fait le point sur :

- les mécanismes de réticulation;

- la structure et la morphologie des systèmes réticulés et les paramètres qui les influencent ;

- les relations entre la structure et les propriétés.

Seules les résines époxy réticulées par des polyamines sont étudiées dans cet article de synthèse. En effet, celles-ci peuvent avoir à la fois une température de transition vitreuse assez élevée $\left(150{ }^{\circ} \mathrm{C}-200{ }^{\circ} \mathrm{C}\right)$, une bonne résistance aux produits chimiques et pétroliers courants et une mise en œuvre aisée. Elles peuvent donc être des solutions pour les applications structurales en température et en présence de fluides corrosifs.

\section{COMPOSANTS DE BASE DES RÉSINES ÉPOXY}

\subsection{Les prépolymères époxy}

Le premier prépolymère époxy apparu sur le marché fut le diglycidyléther de bisphénol A (DGEBA). C'est encore de loin le plus répandu puisqu'il constitue à lui seul $95 \%$ du tonnage mondial. Sa structure chimique est présentée dans la figure 1 . 


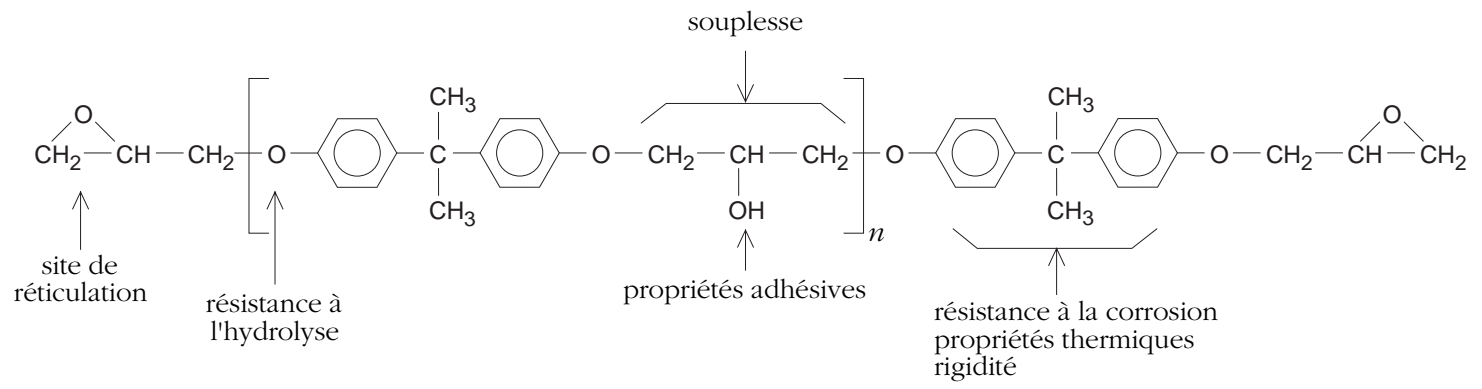

Figure 1

Formule développée du diglycidyléther de bisphénol A (DGEBA).

Structural formula of diglycidylether of bisphenol A (DGEBA).

- $n$ est le degré de polymérisation ;

- la masse molaire du prépolymère est notée $M_{n}$ $(\mathrm{g} / \mathrm{mol})$, avec $M_{n}=340+248 \cdot \mathrm{n}$;

- l'indice d'époxydes (IE) est le nombre d'équivalents d'époxydes, c'est-à-dire de moles d'époxydes, contenues dans un kilogramme de prépolymère (eq $/ \mathrm{kg}$ ou $\mathrm{mol} / \mathrm{kg}$ ), avec $I E=2000 / M_{n}$;

- le poids équivalent d'époxydes $(E E)$ est la quantité de résine en grammes contenant un équivalent d'époxydes.

Pour synthétiser le DGEBA, une certaine quantité de diphénylolpropane (bisphénol A) réagit avec l'épichlorhydrine, en présence de soude. La réaction globale est représentée schématiquement dans la figure 2 .

Il y a formation de chlorure de sodium et départ d'eau. La soude joue un double rôle. Elle est utilisée comme catalyseur pour l'ouverture du cycle époxy de l'épichlorhydrine par le groupe hydroxyle du diphénylolpropane, et comme agent de déshydrohalogénation pour la conversion de l'halohydrine en groupe époxy.

Le degré de polymérisation dépend du rapport molaire entre l'épichlorhydrine et le diphénylolpropane. Suivant sa valeur, la molécule est plus ou moins longue, et la viscosité du produit en est alors modifiée. Ainsi, à température ambiante :

- si $0<n<1 \quad$ : le prépolymère est liquide ;

- si $1<n<1,5$ : le prépolymère est très visqueux ;

- si $n>1,5 \quad$ : le prépolymère est sous forme solide.

Cette synthèse en une étape est appelée procédé Taffy. Il existe un autre procédé, en deux étapes, appelé procédé Avancer; après avoir synthétisé le DGEBA de degré de polymérisation $n=0$, la réaction est avancée<smiles>CC[C@@H](OC)Oc1ccc(C(C)(C)c2ccc(OCC(O)CC(C)Oc3ccc(C(C)(C)c4ccc(OCC5CO5)cc4)cc3)cc2)cc1</smiles>

Figure 2

Synthèse des prépolymères diépoxy DGEBA par le procédé Taffy [3].

Synthesis of DGEBA diepoxy prepolymers by the Taffy process [3]. 
en faisant réagir ce dernier avec du bisphénol A jusqu'à l'obtention de la masse molaire souhaitée. Dans le cas du procédé Avancer, seuls des prépolymères de degré de polymérisation pairs sont synthétisés, alors qu'avec le procédé Taffy, des degrés de polymérisation pairs ou impairs peuvent être obtenus.

Bien que le prépolymère DGEBA occupe la quasitotalité du marché, il existe d'autres prépolymères époxy, différents par leur fonctionnalité (nombre de groupes époxy) ou par la nature chimique des liens entre fonctions époxy réactives, et qui peuvent présenter un intérêt dans des cas particuliers. Deux exemples (le DGEBU et le TGDDM) sont illustrés dans la figure 3.

Les prépolymères époxy ne contiennent pas seulement des oligomères à fins de chaînes époxy. Ces produits secondaires peuvent avoir une grande influence sur les réactions de durcissement ultérieures. De nombreux auteurs [4] se sont attachés à les caractériser, en général par les méthodes chromatographiques : chromatographie par perméation de gel (CPG), chromatographie liquide (CLHP) ou chromatographie sur couche mince, le plus souvent couplées à d'autres techniques telles que la spectrométrie de masse, l'infrarouge ou la résonance magnétique nucléaire.

Les produits secondaires peuvent également être des composés résiduels de réaction. Pour les degrés de polymérisation élevés, des ramifications apparaissent, issues de la réaction d'addition des fonctions époxy sur des hydroxyles.

\subsection{Les durcisseurs polyamines}

Les prépolymères époxy présentent la particularité de pouvoir réagir avec de nombreux groupements fonctionnels, dont les amines, les anhydrides, les mercaptans, les isocyanates, les phénols, etc. Les réactions avec des amines sont les plus couramment utilisées ; ces amines sont présentes dans des polyamines de plusieurs familles : aliphatiques, cycloaliphatiques ou aromatiques. Elles se distinguent par leur fonctionnalité (nombre de groupements amines) et par la nature chimique des liens entre fonctions amines réactives. Quelques exemples sont donnés ci-dessous :

- le diéthylène triamine (DTA), une polyamine aliphatique de formule chimique :

$$
\mathrm{NH}_{2}-\left(\mathrm{CH}_{2}\right)_{2}-\mathrm{NH}-\left(\mathrm{CH}_{2}\right)_{2}-\mathrm{NH}_{2}
$$

- l'isophorone diamine (IPD), une diamine cycloaliphatique primaire de formule chimique :<smiles>CC1(C)CC(N)CC(CN)C1</smiles>

- la 4,4'-diaminodiphénylsulfone (DDS), une diamine aromatique primaire rigide de formule chimique :<smiles>Nc1ccc([Se]c2ccc(N)cc2)cc1</smiles>

- l'hexaméthylène diamine (HMDA), une diamine aliphatique primaire flexible de formule chimique :

$$
\mathrm{NH}_{2}-\left(\mathrm{CH}_{2}\right)_{6}-\mathrm{NH}_{2}
$$

\section{RÉTICULATION}

Lors de la réticulation, les résines thermodurcissables deviennent infusibles et insolubles par réactions chimiques exothermiques (réaction de polyaddition

- le diglycidyléther du 1,4-butanediol (DGEBU) est un prépolymère diépoxy flexible de formule chimique :

$$
\mathrm{CH}_{2}-\mathrm{CH}-\mathrm{CH}_{2}-\mathrm{O}-\left(\mathrm{CH}_{2}\right)_{4}-\mathrm{O}-\mathrm{CH}_{2}-\mathrm{CH}-\mathrm{CH}_{2}
$$

- le tétraglycidyléther du 4,4'-diaminodiphénylméthane (TGDDM) est un prépolymère tétraépoxy rigide de formule chimique :

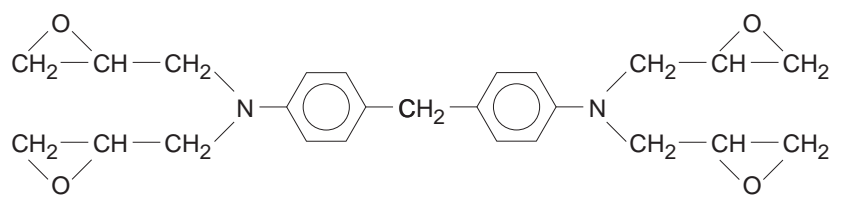

Figure 3

Exemples de prépolymères époxy.

Some epoxy prepolymers. 
irréversible). Le matériau résultant est un réseau tridimensionnel infini. La plupart des formulations nécessitent un apport de chaleur pour réticuler. Une représentation schématique en deux dimensions de la réticulation d'une résine thermodurcissable est donnée en figure 4.

\subsection{Mécanismes de réticulation des prépolymères époxy par des durcisseurs amines}

La réaction d'une amine primaire avec des groupes époxy conduit à plusieurs possibilités. La structure du réseau formé lors de la réticulation est influencée par les vitesses relatives des différentes réactions.

\subsubsection{Réactions (1) et (2)}

La réaction d'un groupe époxy avec une amine primaire produit un alcool secondaire et une amine secondaire (réaction (1)). L'amine secondaire réagit à son tour avec un groupe époxy pour donner une amine tertiaire et deux alcools secondaires (réaction (2)). À chaque réaction entre un époxyde et un hydrogène d'amine, il y a formation d'un groupement hydroxypropyléther. Ces séquences jouent un rôle primordial sur les propriétés du matériau à température ambiante (cf. § 5, fig. 5).

La réactivité des amines dépend de leur basicité, les amines aliphatiques étant les plus réactives et les amines aromatiques les moins réactives. Les réactions (1) et (2) se font suivant le même mécanisme. Cependant, les groupements amines secondaires étant plus encombrés stériquement que les groupements amines primaires, les constantes de vitesse des réactions (1) et (2) peuvent êtres différentes. Les valeurs du rapport $k_{2} / k_{1}$ données dans la littérature sont comprises entre 0,1 et 1,0 [6-9] (tableau 1). (a)

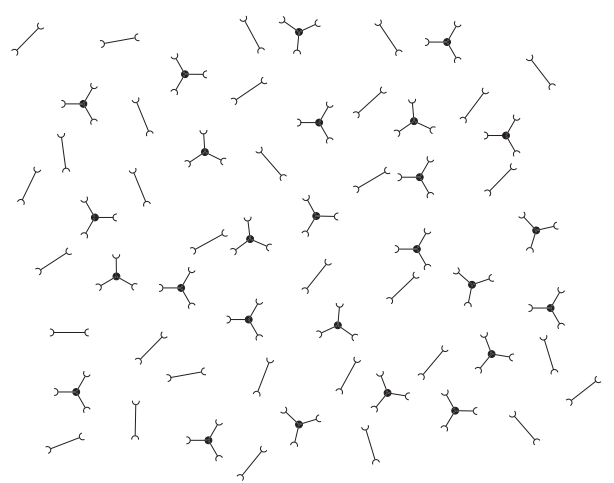

(c)

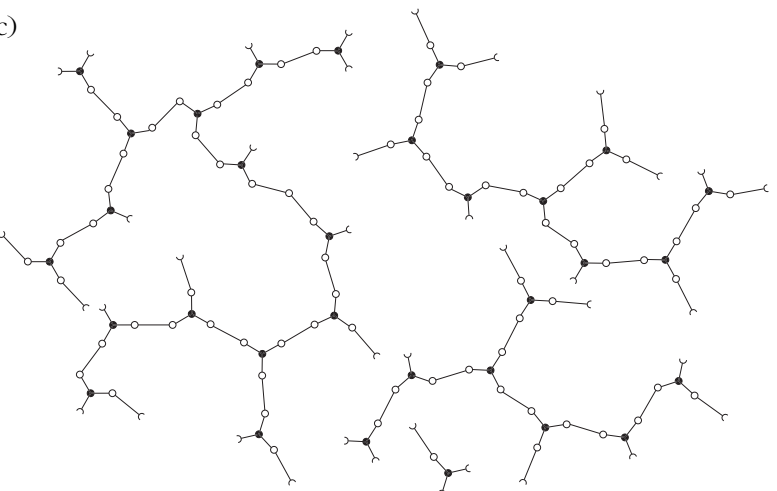

(b)

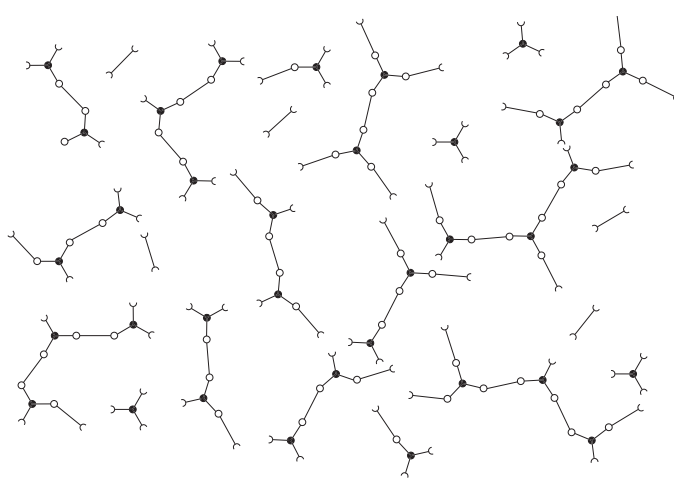

(d)

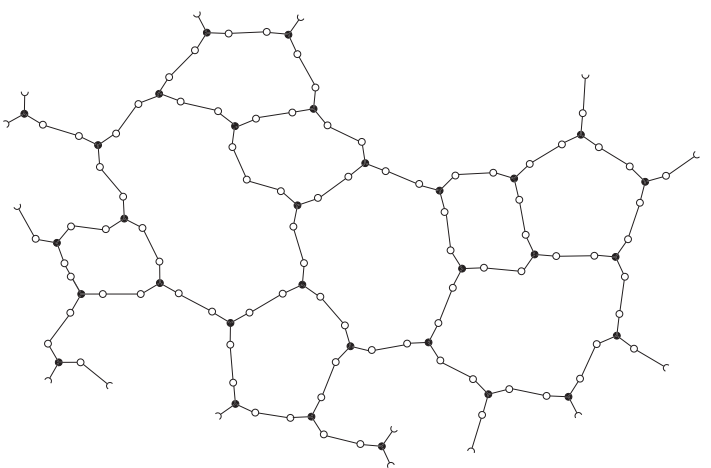

Figure 4

Représentation en deux dimensions de la réticulation d'un polymère thermodurcissable [5].

(a) composants n'ayant pas réagi ; (b) croissance linéaire et branchement des chaînes ; (c) formation d'un gel ; (d) produit complètement réticulé.

Bidimensional representation of crosslinking steps of a thermosetting polymer [5].

(a) unreacted components; (b) linear growth and chain branching; (c) gel formation; (d) fully crosslinked product. 


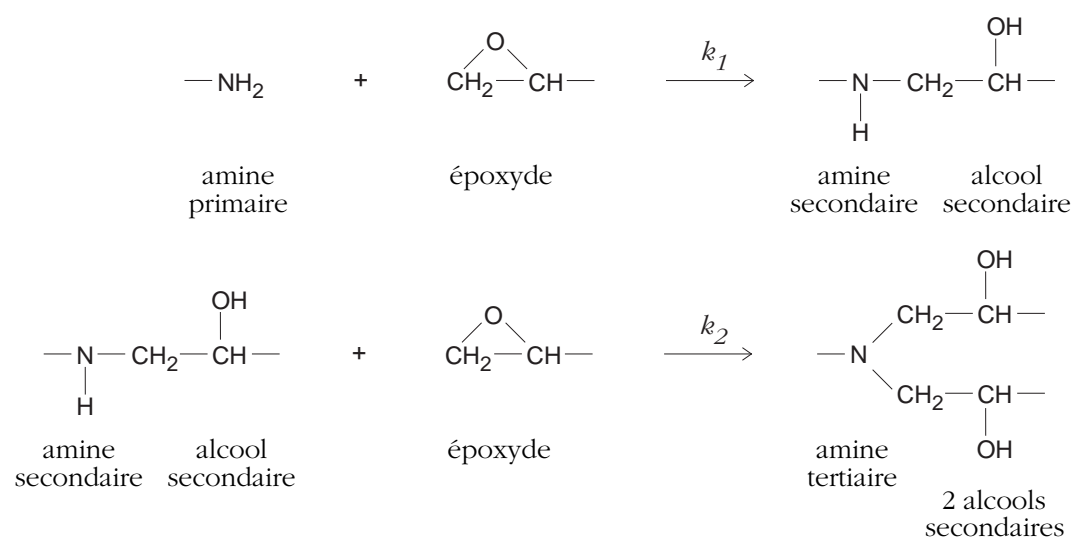

Figure 5

Principales réactions chimiques ayant lieu lors de la réticulation.

Chemical reactions taking place during curing.

\section{TABLEAU 1}

Rapport $k_{2} / k_{1}$ pour différentes amines

$k_{2} / k_{1}$ ratio for different amines

\begin{tabular}{l|c}
\hline & $k_{2} / k_{1}$ \\
\hline Amines aliphatiques & $0,8-1,2$ \\
Amines cycloaliphatiques & $0,5-0,8$ \\
Amines aromatiques & $0,2-0,4$ \\
\hline
\end{tabular}

Quand les réactivités des amines primaires et secondaires sont égales, le branchement se fait dès le début de la réaction. C'est le mécanisme de réticulation statistique; des chaînes de type II (fig. 6) se forment dès le début de la réticulation. Quand les amines primaires sont plus réactives que les amines secondaires, il y a d'abord croissance de chaînes linéaires (type I), puis branchement en chaînes de type II. La différence entre les deux mécanismes apparaît pour des mélanges riches en amines, quand le rapport du nombre d'hydrogènes d'amines sur le nombre d'époxydes est supérieur à 1,5. Dans ces conditions, le mécanisme de réticulation statistique prévoit des structures fortement branchées, alors que le mécanisme de réticulation en deux étapes prévoit principalement des chaînes linéaires [10].

L'effet de la température de cuisson sur le rapport $k_{2} / k_{1}$ a rarement été étudié. Il est considéré comme

chaîne de type I

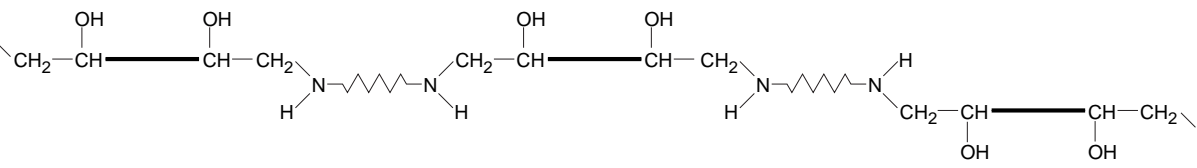

chaîne de type II

Figure 6

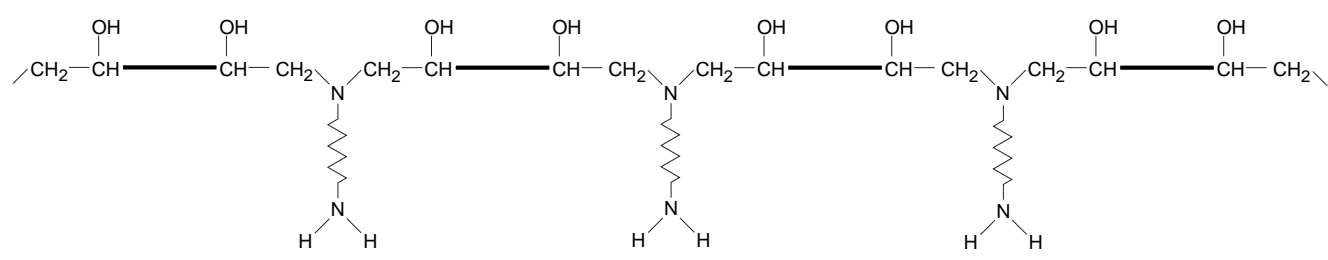

Types de chaînes formées lors de la réticulation d'un système «prépolymère diépoxy/durcisseur diamine primaire » [10].

Types of chains created during the crosslingking of a "diepoxy prepolymer/primary diamine hardener" system [10]. 
indépendant de la température dans la plupart des études cinétiques. Wang et al. [9] ont cependant montré que le rapport $k_{2} / k_{1}$ augmente avec la température de réticulation. La structure et la morphologie, et donc les propriétés chimiques et physiques des systèmes réticulés, peuvent alors varier en fonction de la température de cuisson utilisée.

L'addition de composés contenant des groupes hydroxyles (eau, alcools, phénols, acides) augmente considérablement l'interaction entre les époxydes et les amines ou autres réactifs nucléophiles. La réticulation des prépolymères époxy par des durcisseurs amines a donc un caractère autocatalytique, dû à l'accumulation des groupes hydroxyles durant la réaction.

\subsubsection{Réaction (3)}

Cette réaction correspond à l'homopolymérisation des groupes époxy, catalysée par les amines tertiaires formées par la réaction (2). Comme ces amines tertiaires sont encombrées stériquement, ces réactions sont peu probables en l'absence de catalyseur (fig. 7).

Pour des systèmes contenant un excès de fonctions époxy, cette réaction d'éthérification a lieu une fois tous les hydrogènes d'amines consommés. Pour des systèmes stœchiométriques ou contenant un excès de fonctions amines, l'importance de la réaction (3) par rapport aux réactions (1) et (2) dépend de la nature de l'amine et de la température de réticulation, mais elle est le plus souvent négligeable. Pour des amines aromatiques, à cause de la faible réactivité des amines secondaires par rapport aux amines primaires et des hautes températures nécessaires pour réticuler, la réaction (3) n'est pas toujours négligeable. Cependant, l'éthérification n'a que très rarement été mise en évidence [11-15]. Apparemment, seul le système TGDDM/DDS conduit à la formation d'éthers, car les constantes de vitesse des réactions (2) et (3) sont égales et dix fois plus faibles que la constante de vitesse de la réaction (1) [11]. Ceci est dû au fait que le TGDDM possède dans sa structure chimique des amines tertiaires qui peuvent catalyser l'homopolymérisation.

\subsection{Diagrammes TTT}

Les diagrammes TTT (Temps Température Transformation) sont très utiles pour illustrer les phénomènes ayant lieu lors de la réticulation (gélification, vitrification, réticulation complète et dégradations). Ils ont été développés par Gillham et al., par analogie avec les métaux. Pour différentes températures de cuissons isothermes, les temps d'apparition des différents phénomènes sont mesurés, par exemple, par analyse viscoélastique. L'allure d'un diagramme TTT est donnée en figure 8. Il illustre les principales caractéristiques du processus de réticulation (les températures critiques, les phénomènes ayant lieu lors de la réticulation, les états du matériau). Les diagrammes TTT peuvent être étendus pour inclure la viscosité, l'avancement de la réaction de réticulation, ou une séparation de phase dans le cas de résines modifiées par des élastomères.

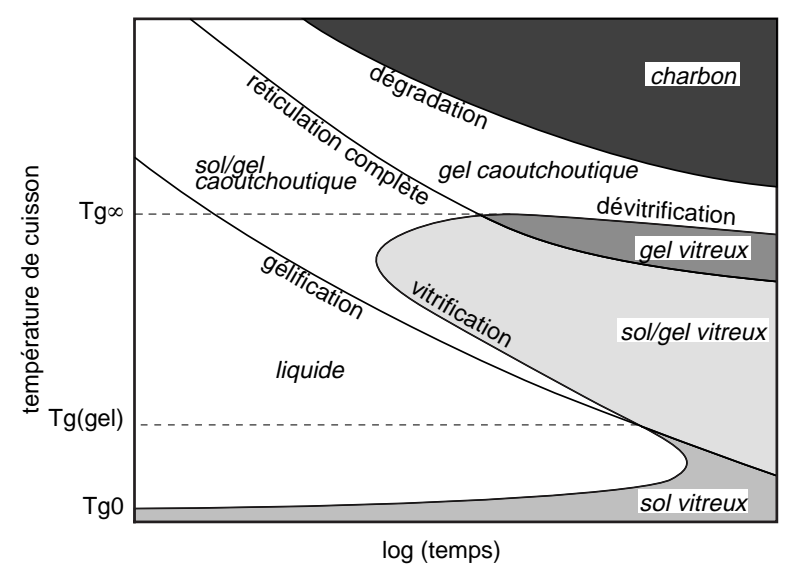

Figure 8

Diagramme TTT montrant les températures critiques et les différents états du polymère [16].

TTT diagram, showing the critical temperatures and the states of the polymer [16].

\begin{tabular}{|c|c|c|c|c|c|}
\hline $\begin{array}{c}\mathrm{OH}^{\mathrm{H}} \\
-\mathrm{CH}-\end{array}$ & + & $\mathrm{CH}_{2}-\mathrm{O}-$ & $\stackrel{k_{3}}{\longrightarrow}$ & $\begin{array}{l}\mathrm{O}-\mathrm{Cr} \\
-\mathrm{CH}-\end{array}$ & $\mathrm{H}_{2}-\mathrm{CH}-$ \\
\hline $\begin{array}{c}\text { alcool } \\
\text { secondaire }\end{array}$ & & époxyde & & éther & $\begin{array}{c}\text { alcool } \\
\text { secondaire }\end{array}$ \\
\hline Figure 7 & & & & & \\
\hline
\end{tabular}




\subsubsection{Les températures critiques}

- Tg0 est la température de transition vitreuse des composants n'ayant pas réagi. En dessous de cette température, le système est en principe non réactif.

- $\operatorname{Tg}$ (gel) est la température à laquelle la gélification et la vitrification coïncident. Entre $\mathrm{Tg} 0$ et $\mathrm{Tg}(\mathrm{gel})$, le système vitrifie avant de gélifier, ce qui bloque en principe les réactions chimiques et empêche la gélification.

- Tgळ est la température de transition vitreuse maximale que le système peut atteindre.

\subsubsection{La gélification}

C'est la transformation irréversible d'un liquide visqueux en un gel viscoélastique. Avant gélification, le système est fusible et soluble. Après gélification, une fraction insoluble (fraction gel) et une fraction soluble (fraction sol) coexistent. La gélification est caractérisée par l'apparition d'un réseau macromoléculaire infini. La viscosité devenant très rapidement infinie à la gélification, le polymère ne peut plus être mis en forme. La gélification apparaît pour un avancement de la réaction chimique de réticulation défini et calculable, $\alpha_{\mathrm{gel}}$. Il dépend de la fonctionnalité, de la réactivité et de la proportion des réactifs. Il est généralement compris entre 0,55 et 0,8 [17]. La gélification ne gêne pas le processus de réticulation (la vitesse de réticulation reste inchangée) et ne peut donc pas être détectée par des techniques sensibles seulement à la réaction chimique, comme l'analyse calorimétrique différentielle.

\subsubsection{La vitrification}

C'est la transformation réversible d'un liquide visqueux ou d'un gel viscoélastique en un solide viscoélastique vitreux. Elle apparaît quand la température de transition vitreuse du gel en croissance devient égale à la température de cuisson. Elle est la conséquence de la diminution de mobilité des chaînes moléculaires liée à l'augmentation de la masse molaire. La vitesse des réactions devient très lente, mais elle n'est pas nulle [18]. La cinétique n'est plus contrôlée par la chimie du système mais par la diffusion. L'avancement à la vitrification augmente avec la température de cuisson. Afin de poursuivre la réticulation, il est possible de dévitrifier le matériau en augmentant la température de cuisson au-dessus de la température de transition vitreuse du réseau.

\subsubsection{La réticulation complète}

La courbe de réticulation complète représente le temps nécessaire, pour chaque température de cuisson, pour que $\mathrm{Tg}$ atteigne $\mathrm{Tg} \infty$. Le réseau est alors considéré entièrement construit, bien qu'il existe toujours un certain nombre de fonctions n'ayant pu réagir.

\subsubsection{Les dégradations}

Aux hautes températures, la dégradation thermique devient importante, et peut empêcher la réticulation complète de se faire. Deux types de dégradations interviennent : la dévitrification puis la revitrification. La dévitrification entraîne la formation d'un gel caoutchoutique. Elle correspond à une diminution de $\mathrm{Tg}$ (et de la rigidité) qui passe en dessous de la température de cuisson à cause de la diminution de la densité de réticulation et/ou de la formation de plastifiants. La revitrification entraîne la formation de charbon. Elle correspond à une augmentation de $\mathrm{Tg}$ (et de la rigidité) à cause d'une densification ou de la volatilisation de plastifiants de faible masse molaire.

\subsection{Importance du cycle de cuisson}

Lors de la confection de pièces de grands volumes, la masse qui entre en réaction joue un rôle essentiel $[3,19$, 20]. En effet, en réticulant, les molécules cèdent leur chaleur de réaction aux molécules voisines. Comme la température ne peut s'équilibrer instantanément et que la résine est mauvaise conductrice de la chaleur, il se produit un processus d'emballement qui a son maximum d'intensité vers le centre de la pièce. Pour éviter ce phénomène, une cuisson en plusieurs étapes est souvent adoptée : gélification à température modérée, augmentation par paliers de la température de durcissement (idéalement, il faudrait suivre la courbe de vitrification du diagramme TTT) et finalement, postcuisson à température supérieure à $\mathrm{Tg} \infty$.

Le refroidissement en fin de cuisson doit aussi être soigneusement contrôlé. Il doit être très lent, en particulier au voisinage de la température de transition vitreuse, afin d'éviter les contraintes internes susceptibles de créer des amorces de fissures, notamment dans le cas de pièces massives avec inserts métalliques. Contrôler la cuisson et le refroidissement permet aussi de maitriser le retrait au moulage, cependant faible pour les résines époxy $(0,5 \%-1 \%)$. 


\section{STRUCTURE ET MORPHOLOGIE}

\subsection{Structure}

$\mathrm{Si}$ un système stœchiométrique, par exemple DGEBA $(n=0) /$ DDS réticule complètement, sans réaction secondaire, une maille d'un réseau idéal peut alors être représentée, en deux dimensions, par le schéma de la figure 9 .

À chaque fois qu'une amine (primaire ou secondaire) réagit avec un groupement époxy, il y a formation d'une séquence hydroxypropyléther. Ces séquences sont responsables des mouvements moléculaires locaux qui jouent un rôle primordial sur les propriétés des réseaux à l'état vitreux (cf. § 5). Les réseaux sont caractérisés par $M_{c}$, la masse molaire moyenne des segments de chaînes entre points de réticulation ou par $\xi_{M}$, leur densité de points de réticulation, dont le calcul théorique est décrit dans la littérature pour des systèmes totalement réticulés [21].

Toutefois, le réseau idéal n'existe pas. Il y a toujours, dans un réseau réel (fig. $4 \mathrm{~d}$ ), une distribution de la taille des mailles, même lorsque le mélange est stœchiométrique, que le réseau est entièrement construit et que les composants de base sont parfaitement purs et monodispersés. Il peut aussi y avoir un certain nombre d'imperfections dans les réseaux :

- une distribution de la taille des mailles due à la distribution des masses molaires du prépolymère diépoxy ;
- des chaînes pendantes, inactives mécaniquement, dues à des fins de chaînes anormales sur les composants de base ou à un excès de l'un des composants de base, ou bien dans le cas de systèmes catalysés ;

- des nœuds de réticulation supplémentaires dus à la formation de liens éthers dans des mélanges catalysés ou contenant un excès de groupes époxy ;

- des chaînes de type I (fig. 6) dans les systèmes contenant un excès de durcisseur ;

- des nœuds physiques de réticulation, dus aux enchevêtrements de chaînes lors de la création du réseau ;

- des petites boucles pendantes, inactives mécaniquement, dues à des réactions intramoléculaires des groupes fonctionnels. La probabilité de formation d'une petite boucle inactive dépend de sa stabilité thermodynamique.

\subsection{Morphologie}

\subsubsection{Topographie des réseaux}

De nombreuses propriétés des réseaux dépendent non seulement des caractéristiques structurales, mais aussi de leur répartition spatiale, c'est-à-dire de la topographie du réseau. Celle-ci est difficilement accessible expérimentalement et c'est sur la base d'un modèle physique de formation du réseau qu'elle peut être évaluée. Deux approches ont été développées.

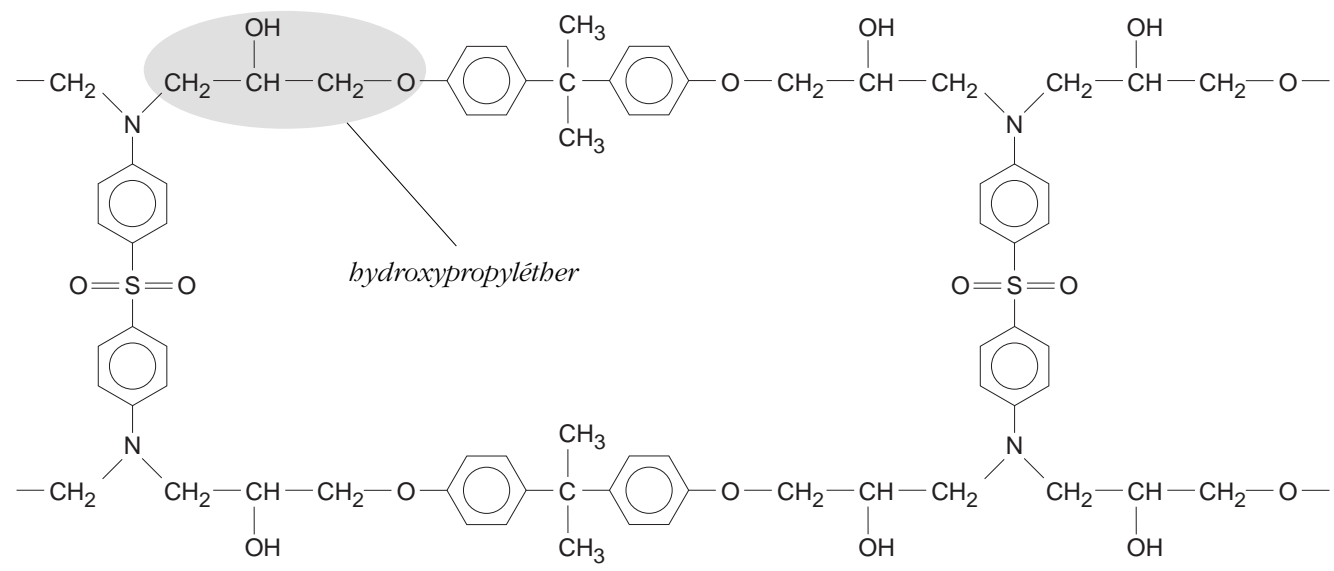

Figure 9

Représentation schématique en deux dimensions d'une maille d'un réseau idéal.

Schematic representation in two dimensions of an ideal network unit cell. 
L'une, proposée par Dusek [22], est fondée sur un modèle de réseau à nœuds et segments orientés aléatoirement à partir de ces nœuds. L'autre, développée par Topolkaraev, Berlin et Oshmyan [23], utilise une simulation informatique de la structure du réseau, assimilé à un maillage régulier. Ces deux approches sont statistiques et diffèrent principalement au niveau mathématique. Elles soulignent des détails différents de la structure du réseau mais conduisent aux mêmes conclusions :

- La topographie des réseaux n'est pas très uniforme ; il existe une large distribution de taille des mailles ainsi que des nœuds de réticulation de différente connectivité. Cette distribution est le résultat de la statistique de réaction.

- La réticulation n'atteint jamais l'avancement total, à cause de l'isolement topographique de groupes réactifs durant la réaction de réticulation statistique.

Le module élastique des réseaux à l'équilibre peut être calculé en prenant en compte la distribution topographique du réseau et l'élasticité vraie d'une chaîne entre nœuds de réticulation [23]. Il y a bon accord entre le module caoutchoutique ainsi calculé et le module caoutchoutique expérimental pour différents types de systèmes « époxydes/amines ».

\subsubsection{Morphologie nodulaire}

Une abondante littérature existe sur la morphologie nodulaire des résines époxy, par exemple, [24] et [25]. Pour certains, il existerait des hétérogénéités spatiales de densité de réticulation. Les résines époxy pourraient être constituées de nodules de forte densité de réticulation baignant dans une matrice de plus faible densité de réticulation. La taille des nodules serait comprise entre 5 et $100 \mathrm{~nm}$ et dépendrait des conditions de polymérisation telles que la concentration en durcisseur [24] et 26], la cuisson [24] et [26], ou l'agitation lors du mélange [27]. Les techniques ayant été utilisées pour mettre en évidence cette morphologie sont les suivantes : la microscopie électronique à transmission et à balayage, la diffraction des rayons $\mathrm{X}$ aux petits angles, la résonance paramagnétique électronique, la résonance magnétique nucléaire et la spectrométrie mécanique [28] et [29].

L'existence d'une telle morphologie est cependant très discutée ; certains auteurs considèrent qu'elle n'est qu'apparente et résulte du mode de préparation des échantillons [22] et [30]. Aujourd'hui, la plupart se sont ralliés à ce point de vue. Toutefois, il se pourrait que cette morphologie apparaisse lors du passage de la transition vitreuse en refroidissant le matériau et soit la conséquence d'un «gel» d'une configuration particulière. Cette morphologie pourrait alors disparaître dès que la température dépasserait de nouveau la température de transition vitreuse.

\section{RELATIONS ENTRE LA STRUCTURE ET LES PROPRIÉTÉS À LA TRANSITION VITREUSE ET À L'ÉTAT CAOUTCHOUTIQUE}

Afin d'étudier les relations entre la structure et les propriétés des résines époxy réticulées par des polyamines, les auteurs ont cherché à modifier la structure du réseau. Pour cela, plusieurs voies ont été suivies :

- en modifiant la flexibilité des composants de base [13, 14, 21, 29, 31-34] ;

- en variant l'avancement de la réticulation, en jouant sur la température ou la durée de cuisson [35-40] ;

- en changeant les proportions du mélange [15, 21, 25, 36-38, 41-46] ;

- en modifiant la longueur du prépolymère époxy [37, 44, 47-50] ;

- en utilisant des extenseurs de mailles de même structure chimique que le durcisseur [13, 21, 29, 31$34,51]$;

- en utilisant un catalyseur [12].

L'analyse du comportement de ces résines a été effectuée dans trois gammes de températures : (1) au voisinage de $\mathrm{Tg}$, (2) à l'état caoutchoutique $(\mathrm{T}>\mathrm{Tg}$ ) et (3) à l'état vitreux, entre $T_{\beta}$, et $\mathrm{Tg}$, soit pour les températures usuelles d'utilisation. Les résultats obtenus pour les domaines (1) et (2) de températures sont présentés dans ce chapitre, le domaine (3) faisant l'objet du chapitre suivant.

\subsection{La région de la transition vitreuse}

L'influence de la structure du réseau sur le phénomène de transition vitreuse est bien décrit dans la littérature. Les principaux résultats sont les suivants :

\begin{tabular}{l|l} 
Paramètre & Effet sur Tg \\
\hline Stoechiométrie & passe par un maximum \\
Avancement de la réticulation & \\
Rigidité des constituants & \\
de base & \\
Taille des mailles & \\
Utilisation d'un catalyseur &
\end{tabular}


Des expressions mathématiques ont été proposées pour rendre compte de la température de transition vitreuse des réseaux époxy. Elles ont en commun de se déduire de la température de transition vitreuse théorique du copolymère linéaire homologue, $\mathrm{Tg}_{\text {lin }}$, et de la densité de points de réticulation. Ces expressions dérivent soit d'un calcul d'entropie configurationnelle (théorie de Di Marzio [52]), soit d'un modèle de volume libre (théorie de Fox et Loshaek [53]). Récemment, Verdu et al. [27, 51, 54] ont proposé une formule prédictive relevant du même esprit, qui est vérifiée avec une précision raisonnable par une quarantaine de systèmes «époxyde/amine».

\subsection{L'état caoutchoutique}

Bien que certaines hypothèses de l'élasticité caoutchoutique ne soient pas vérifiées par les réseaux époxy fortement réticulés, en raison du caractère non gaussien et de la limite d'extensibilité des courtes chaînes du réseau, l'expérience montre que ce modèle simple garde, en pratique, une bonne valeur prédictive pour le calcul du module d'élasticité. Il s'exprime par la formule suivante :

$$
E=\phi \cdot \frac{3 \rho R T}{M_{c}}
$$

avec :

$\rho$ : masse volumique du polymère,

$E$ : module élastique à l'équilibre,

$M_{c}$ : masse molaire moyenne entre points de réticulation,

$T$ : température, $R$, constante des gaz parfaits,

$\phi$ : facteur de front.

Dans quelques cas, $\phi$ peut être prédit à partir de considérations physiques, mais le plus souvent, il s'agit d'un facteur purement empirique. Il est généralement admis que $\phi=1$ pour les systèmes stœechiométriques entièrement construits.

Le module caoutchoutique d'un réseau n'est pas seulement fonction de la densité de réticulation, mais aussi de la flexibilité des mailles des réseaux [29]. L'établissement de relations quantitatives entre le module caoutchoutique et $M_{c}$ est donc loin d'être un problème trivial.

Bien que $E$ et $\mathrm{Tg}$ soient, par leur nature même, des grandeurs physiques bien différentes, leurs valeurs sont gouvernées par les mêmes caractéristiques structurales. En effet, les valeurs de $E$ et $\mathrm{Tg}$ les plus élevées sont observées pour les mélanges stœchiométriques, pour les mélanges les plus réticulés et pour les mélanges les plus rigides. Il existe donc une corrélation entre ces deux caractéristiques.

\section{RELATIONS ENTRE LA STRUCTURE ET LES PROPRIÉTÉS À L'ÉTAT VITREUX ENTRE $T_{\beta}$ ET Tg}

Si les relations entre structure et propriétés dans la région de la transition vitreuse et du plateau caoutchoutique sont bien décrites, il n'en est pas de même à l'état vitreux, c'est-à-dire pour les températures d'utilisation des résines époxy. Beaucoup d'études ont conduit à des résultats divergents ou discutés. Néanmoins, les mouvements moléculaires, mis en évidence par les transitions secondaires et la transition vitreuse, semblent jouer un rôle très important.

\subsection{Les mouvements moléculaires locaux}

Les transitions secondaires, qui se manifestent à l'état solide, sont associées à des possibilités de mouvements moléculaires locaux ou relaxations secondaires. Ces mouvements moléculaires locaux affectent non seulement la réponse viscoélastique, mais aussi les propriétés mécaniques (module élastique, déformation plastique, etc.) ou la diffusion de solvants.

Les transitions secondaires dans les résines époxy sont étudiées depuis plus de vingt-cinq ans, le plus souvent par spectrométrie mécanique. Dès 1967, Dammont et Kwei [55] ont mis en évidence l'existence d'une transition $\gamma$ vers $-125^{\circ} \mathrm{C}$ et d'une transition $\beta$ vers $-60^{\circ} \mathrm{C}$ dans des systèmes «époxyde/amine».

La relaxation $\gamma$ est observée uniquement lorsque le prépolymère est un diglycidyléther de diol aliphatique renfermant au moins deux groupes méthylènes successifs [56] et [57] ou que le durcisseur est une amine aliphatique $\mathrm{NH}_{2}-\left(\mathrm{CH}_{2}\right)_{n}-\mathrm{NH}_{2}$ d'indice $n$ supérieur ou égal à quatre [56]. La relaxation g est donc liée au mouvement de rotation (crankshaft motion) de ces unités aliphatiques particulièrement flexibles autour de l'axe de la chaîne (structure (1)).

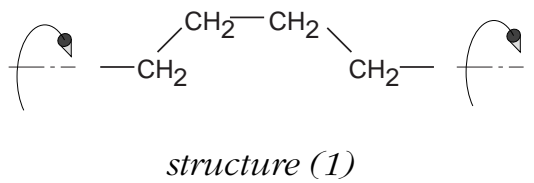


Le couplage entre la spectrométrie mécanique et la RMN ${ }^{13} \mathrm{C}$ haute résolution des solides a permis à Eustache [14] d'attribuer la relaxation $\beta$ au mouvement de vilebrequin des séquences hydroxypropyléther formées lors de la réticulation, par réaction entre les amines et les époxydes (structure (2)).<smiles>CC1(OCC(O)CC2CC2)CCC1</smiles>

structure (2)

La structure en réseau des résines époxy entraîne des effets de coopérativité des mouvements des séquences hydroxypropyléther [29] et [33]. Ainsi, plus le nombre de séquences hydroxypropyléther augmente, plus les mouvements locaux sont intenses. À nombre de séquences égal, l'intensité des mouvements augmente avec la densité de réticulation du réseau et avec la rigidité des constituants de base. La relaxation $\beta$ apparaît à plus haute température que la relaxation $\gamma$ car le groupe hydroxyle latéral entraîne une augmentation de l'énergie nécessaire à l'initiation des mouvements de rotation par rapport à $-\left(\mathrm{CH}_{2}\right)_{n}-$.

\subsection{Influence des mouvements moléculaires locaux sur les modules}

Sur les courbes du module de perte (fig. 10), mesurées par spectrométrie mécanique, la largeur et l'amplitude du pic de la transition $\beta$ croissent avec le degré de réticulation. Les conséquences de la relaxation $\beta$ sur le module de conservation sont illustrées dans la figure 11, dans le cas de l'utilisation d'extenseurs de mailles. À basse température, en dessous de $T_{\beta}$, le module est d'autant plus élevé que la densité de réticulation est importante. Ceci est lié à une compacité plus forte des systèmes les plus réticulés. À température ambiante, audessus de $\mathrm{T}_{\beta}$, le phénomène inverse est observé. L'inversion de l'ordre des modules a lieu au voisinage de $\mathrm{T}_{\beta}$; elle est directement liée à la forme du pic et donc à l'importance des mouvements moléculaires locaux [29].
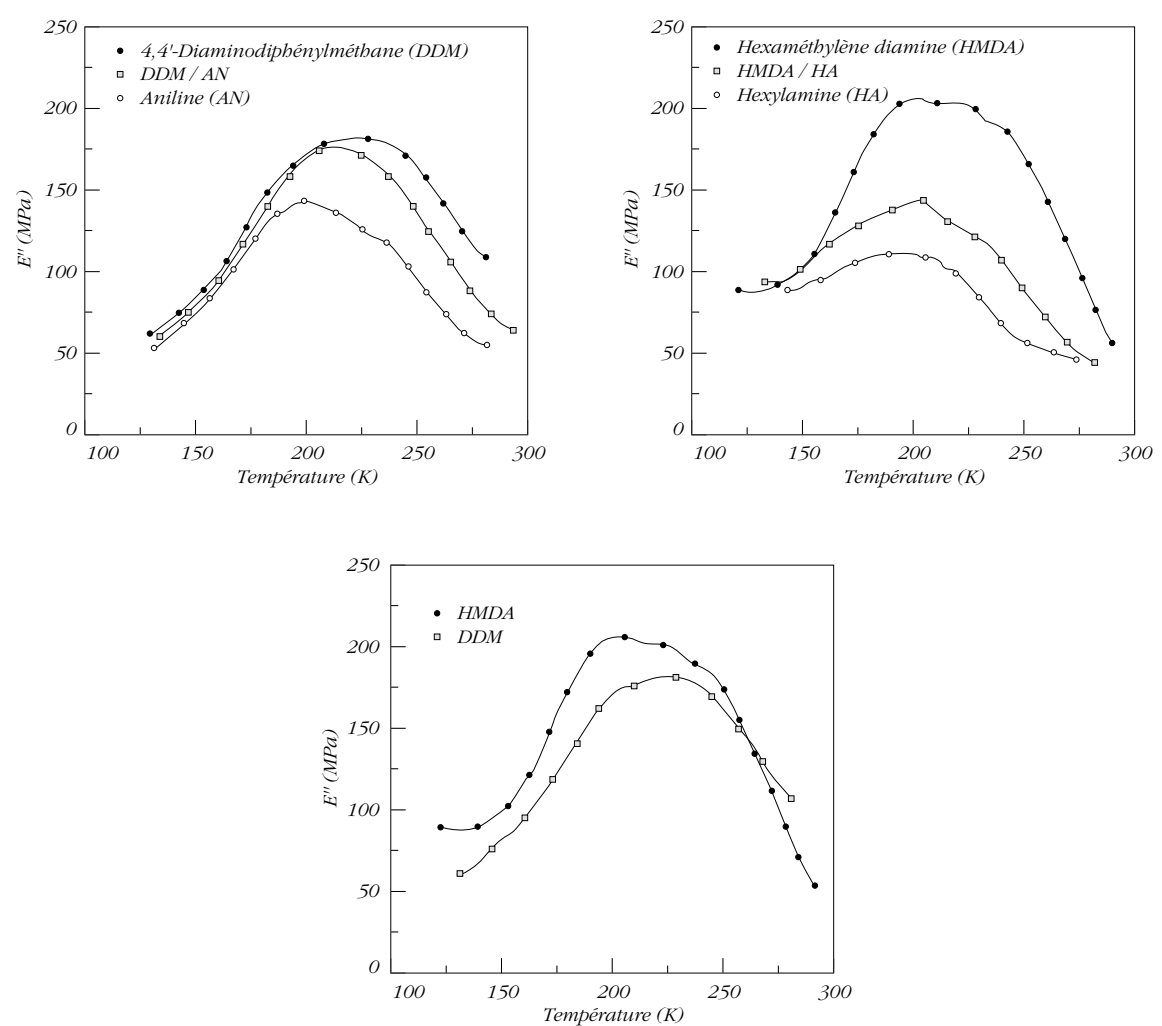

Figure 10

Évolution de $E^{\prime \prime}$ avec la température, à $1 \mathrm{~Hz}$, pour différents systèmes DGEBA/amines.

Loss modulus E" versus temperature, at $1 \mathrm{~Hz}$, of several DGEBA/amine systems. 

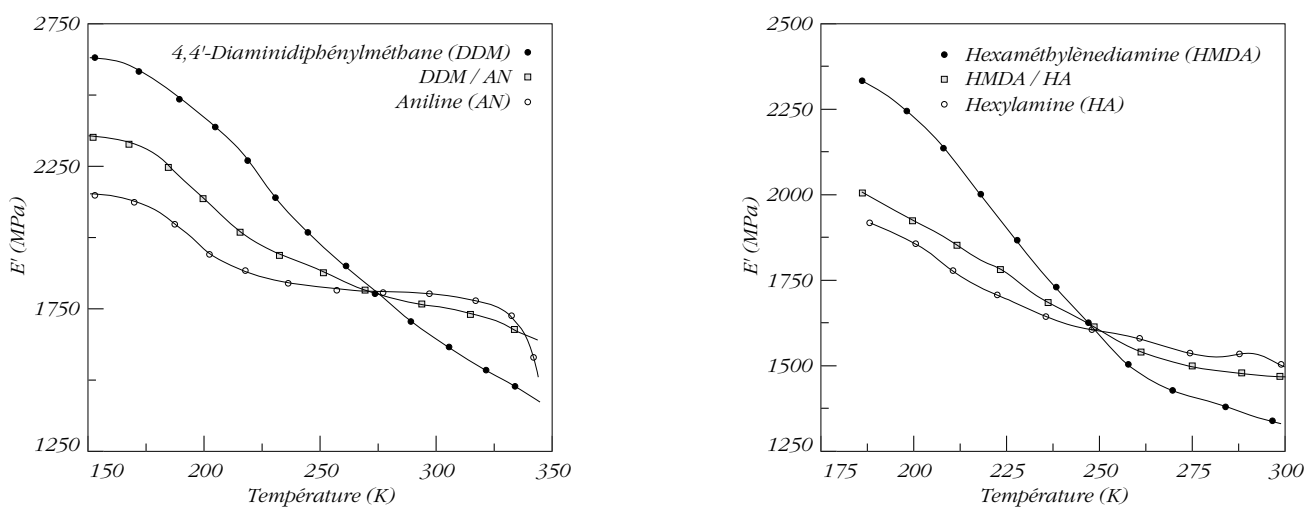

Figure 11

Évolution de E' avec la température, à $1 \mathrm{~Hz}$, pour différents systèmes DGEBA/amines.

Storage modulus E' versus temperature, at $1 \mathrm{~Hz}$, of several DGEBA/amine systems.

Les mouvements responsables de la transition $\beta$ peuvent être inhibés par l'introduction d'additifs de structure chimique appropriée $[21,58,59]$. De tels composés sont dénommés antiplastifiants car ils augmentent la valeur du module vitreux, alors qu'ils jouent le rôle de plastifiant en abaissant la température de transition vitreuse.

\subsection{Influence des mouvements moléculaires locaux sur la déformation plastique}

Escaig et al. [60] ont développé une analyse thermodynamique du comportement dans la zone plastique. Celle-ci montre, qu'à partir d'expériences de compression conduites à vitesse de déformation plastique constante et pour une large gamme de température en dessous de $\mathrm{Tg}$, il est possible de déterminer :

- $\sigma_{Y}$ et $\varepsilon_{Y}$, la contrainte et la déformation au seuil de plasticité ;

- $\Delta G_{a}$, l'enthalpie libre d'activation des mécanismes de déformation plastique ;

- $V_{0}$, le volume d'activation apparent, qui traduit la sensibilité de la déformation de la structure à la contrainte.

Les variations de $\Delta G_{a}$ avec la température, ainsi que la valeur de $V_{0}$, dans le cas du système DGEBA/DDM sont représentées sur la figure 12.

Dans cette approche thermodynamique, l'existence d'un palier signifie que, dans ce domaine de températures, l'activation thermique permet les mécanismes impliqués dans la déformation plastique. Pour le système DGEBA/DDM, un palier apparaît pour des températures correspondant à la transition $\beta$, indiquant que ce sont les mouvements moléculaires associés à cette transition qui sont mis en jeu dans la déformation plastique. Dans le cas de résines époxy possédant dans leur squelette des groupements méthylènes $-\left(\mathrm{CH}_{2}\right)_{n}-$ responsables de la transition $\gamma$, un palier supplémentaire à plus basse température apparaît, si $n$ est suffisamment grand. D'un point de vue qualitatif, la dépendance en température de $\sigma_{Y}$ est la même pour tous les systèmes étudiés.

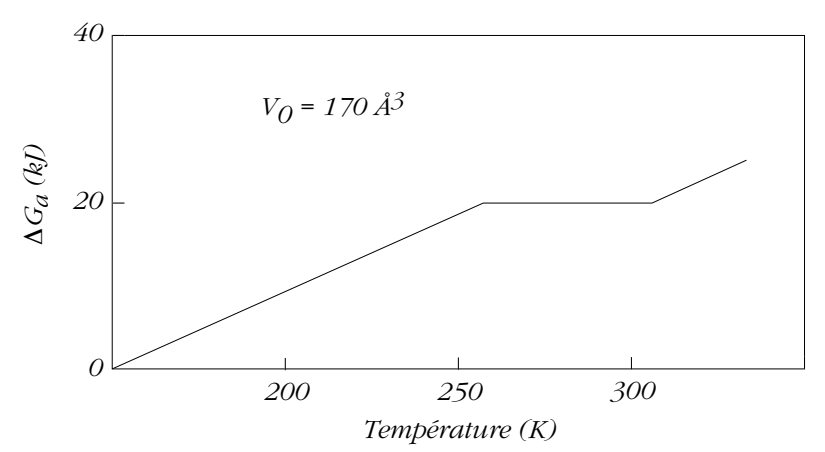

Figure 12

Évolution de $\Delta G_{a}$ avec la température pour le système DGEBA/DDM [58].

Evolution of $\triangle G_{a}$ of DGEBA/DDM versus temperature [58].

La diminution de la densité de réticulation par ajout d'un extenseur de maille entraîne une diminution de $\sigma_{Y}$ à température ambiante, en même temps qu'une diminution de $\mathrm{Tg}$ [60-62]. Cela signifie que les réseaux les plus lâches ont, en première approche, une plus grande aptitude à se déformer plastiquement. En revanche, les valeurs de $\sigma_{Y}$ mesurées à $-196^{\circ} \mathrm{C}$ sont constantes. 
Ceci indique que pour des températures inférieures à $\mathrm{T}_{\beta}$, le mécanisme de déformation gouvernant l'écoulement plastique ne dépend pas de la densité de réticulation.

\subsection{Influence des mouvements moléculaires locaux sur les phénomènes de diffusion de l'eau}

Cukierman [29] et [63] a étudié l'absorption d'eau de quatre réseaux époxy modèles, présentant une variation progressive de densité de points de réticulation et de rigidité de mailles. Le coefficient de diffusion augmente à la fois avec le degré de réticulation et avec la rigidité des mailles, c'est-à-dire avec le nombre de mouvements de type $\beta$. La figure 13 montre qu'une corrélation raisonnable existe entre le coefficient de diffusion de l'eau et l'aire du pic du module de perte de la transition $\beta$. Il a été proposé que les mouvements locaux devaient permettre aux molécules d'eau de cheminer d'un site de mobilité à un autre.

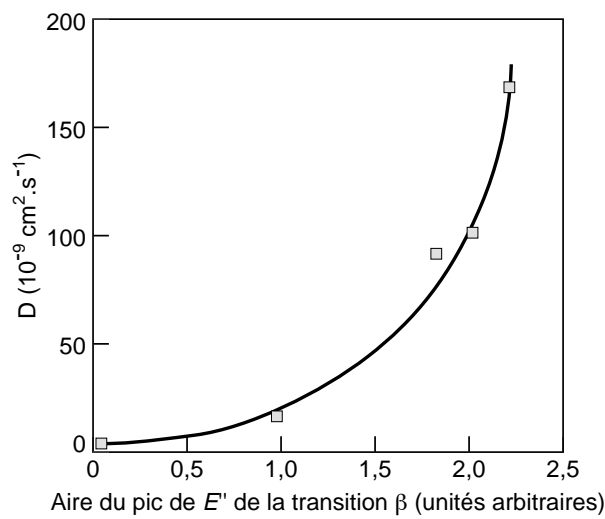

Figure 13

Corrélation entre le coefficient de diffusion de l'eau et l'aire du pic de $E$ "' de la transition $\beta$ [63].

Correlation between the coefficient of diffusion of water and the area under the peak of E" of the $\beta$ transition [63].

\subsection{Influence des mouvements moléculaires sur le coefficient de dilatation thermique}

Bauchière [21] a étudié l'évolution du coefficient de dilatation thermique $\alpha$ du système DGEBA/DDM, pour lequel la densité de réticulation est modifiée en jouant sur les proportions du mélange ou sur l'utilisation d'un extendeur de maille. Les résultats sont présentés dans la figure 14

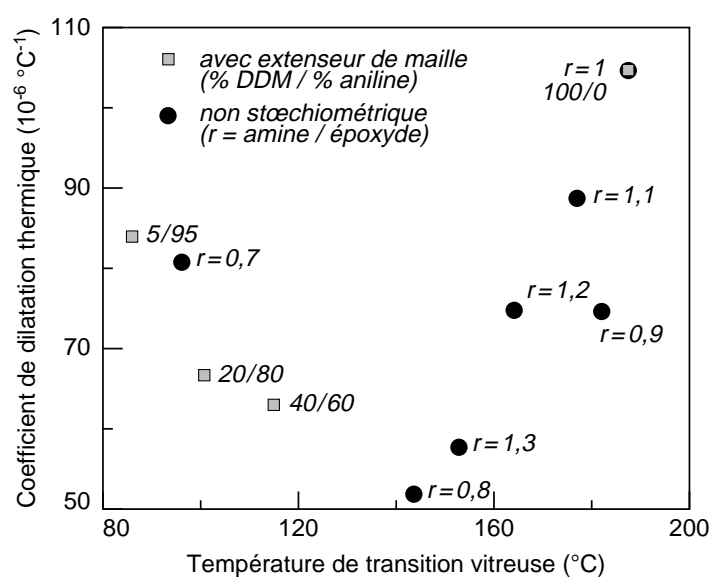

Figure 14

Corrélation entre le coefficient de dilatation thermique et la température de transition vitreuse d'un système DGEBA/ DDM [21]

Correlation between the coefficient of thermal expansion and the glass transition temperature of DGEBA/DDM-based systems [21].

D'après ces résultats, Bauchière a envisagé que les variations du coefficient de dilatation thermique avec la température de transition vitreuse pourraient résulter de deux effets antagonistes, l'un lié à la transition vitreuse et l'autre à la transition $\beta$. Ainsi, il a été montré de façon empirique, que le produit $\alpha$. Tg est presque constant ; les matériaux possédant une température de transition vitreuse faible ont un coefficient de dilatation thermique relativement important. Cette tendance affecterait les systèmes ne possédant pas ou peu de transitions à basses températures, comme les systèmes quasi linéaires ou présentant un fort écart à la stœchiométrie. D'autre part, l'étude du PVC, avec ou sans antiplastifiant, a permis de montrer le rôle de la transition secondaire sur la valeur du coefficient de dilatation à l'état vitreux : les matériaux présentant une transition secondaire importante posséderaient un coefficient de dilatation thermique nettement supérieur à leurs homologues possédant une transition secondaire atténuée. Cet effet affecterait les réseaux denses ou ayant un faible écart à la stœchiométrie. La balance entre les deux facteurs ferait apparaître un minimum.

\subsection{Influence de la masse volumique sur les propriétés des résines époxy}

Il a souvent été observé qu'à température ambiante, la masse volumique d'un système époxy diminue quand la densité de réticulation augmente, lorsque 
celle-ci est contrôlée par la cuisson ou par les proportions du mélange [42, 43, 64-69]. Des auteurs [42, 43, 67, 69-71] ont cherché des explications volumétriques à ce comportement. Ainsi, Gillham et al. [67] et [71] ont suggéré récemment que l'augmentation de la fraction de volume libre avec l'avancement de la réticulation est reliée à un phénomène d'antiplastification. Dans un système partiellement réticulé, les réactifs n'ayant pas réagi ou ayant partiellement réagi, pourraient jouer le rôle d'antiplastifiants en remplissant du volume libre et en bloquant les mouvements moléculaires de l'état vitreux. Ainsi, les systèmes les moins réticulés auraient une masse volumique et un module plus élevé.

En revanche, la diminution de la densité de réticulation par augmentation de la taille des prépolymères [50] ou par l'ajout d'extendeurs de maille [21] entraîne une diminution de la masse volumique. Il n'y a pas, dans ce cas, d'extrémité de chaînes pouvant jouer le rôle d'antiplastifiant. Il est envisageable que la diminution du nombre de points de réticulation tende à augmenter le volume inoccupé dans le réseau. Les chaînes auraient tendance à s'écarter les unes des autres en l'absence des points de réticulation, d'où une diminution de la masse volumique.

Beaucoup d'auteurs ont cherché des explications volumétriques à l'évolution des modules vitreux avec la densité de réticulation. Le module augmenterait avec la masse volumique. Cependant, cette observation n'est pas valide dans le cas de systèmes avec extenseurs de maille ou prépolymères de différentes longueurs. L'explication en termes de mouvements secondaires est donc plus vraisemblable [29].

Kong [69] a mesuré la masse volumique d'un système TGDDM/DDS en fonction de son histoire thermique. Il a suggéré que la diminution de la masse volumique à température ambiante pour les systèmes les plus réticulés pourrait être partiellement due à l'échappement de DDS n'ayant pas réagi et/ou d'autres espèces de faible masse molaire (fig. 15).

Lorsque, au cours de sa mise en œuvre, le polymère est refroidi, celui-ci se «fige » avec un excès de volume spécifique et d'enthalpie. En dessous de Tg, la mobilité résiduelle dans le matériau va permettre une évolution vers l'équilibre. Cette évolution est le vieillissement par relaxation de volume (VRV). L'apparition de la vitrification durant la réticulation entraîne une compétition entre le VRV, qui augmente la masse volumique, et les réactions chimiques de réticulation, qui diminuent la masse volumique, dans le cas où le matériau n'est pas entièrement réticulé [65]. Ce phénomène de VRV n'a pas suscité beaucoup d'attention; il pourrait peut-être expliquer certains résultats discutés de la littérature.

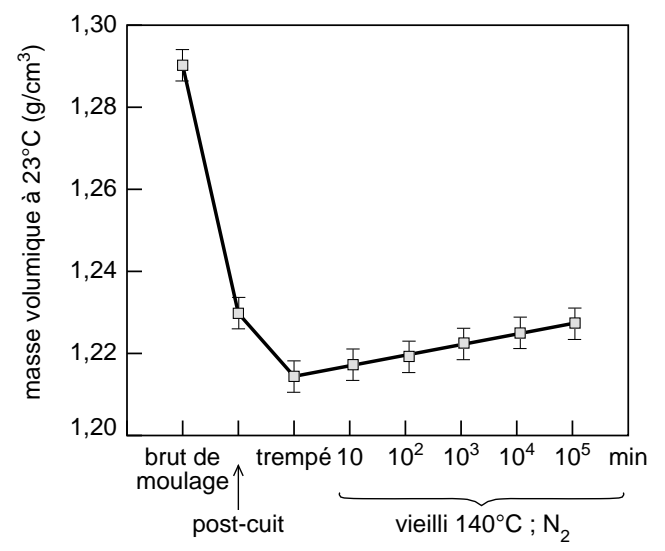

Figure 15

Masse volumique d'un système TGDDM/DDS en fonction de son histoire thermique [69].

Density of TGDDM/DDS versus its thermal history [69].

\subsection{Rupture des résines époxy}

Les énergies de rupture des résines époxy sont de l'ordre de 100 à $300 \mathrm{~J} / \mathrm{m}^{2}$, ce qui représente au moins 100 fois l'énergie nécessaire pour rompre uniquement des liaisons covalentes (moins de $1 \mathrm{~J} / \mathrm{m}^{2}$ ). D'autres processus de dissipation d'énergie doivent donc se produire, notamment en extrémité de fissure. Dans les résines époxy fortement réticulées, des bandes de cisaillement ont pu être observées. En revanche, il y a très peu d'évidence de craquelures, ce qui s'explique par les difficultés de réorientation des chaînes entre nœuds de réticulation.

Des auteurs ont cherché à relier le comportement mécanique à rupture à la transition $\beta$. Dans certains cas [58], la résilience augmente avec la quantité de mouvements $\beta$. Cependant, cette relation n'est pas universelle et reste controversée. Elle semble dépendre du système, de l'auteur ou de la technique utilisée pour faire varier la densité de réticulation : cuisson, proportions du mélange, utilisation d'extenseurs de maille ou de prépolymères de différentes longueurs, etc. Par ailleurs, les tendances obtenues peuvent être inversées selon les conditions dans lesquelles sont réalisés les essais. La figure 16 montre l'effet de la vitesse de sollicitation et de la présence d'un antiplastifiant sur 
l'énergie de rupture d'un système DGEBA/DDM [72]. Dans le cas des essais réalisés à faible vitesse, le système avec antiplastifiant est moins fragile, alors que le contraire est observé pour les vitesses plus élevées.

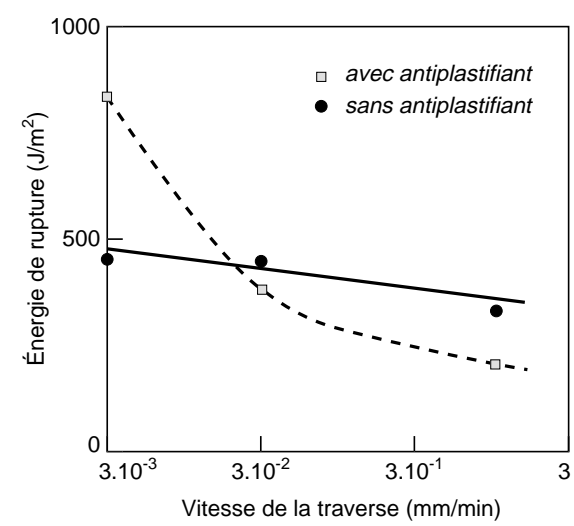

Figure 16

Énergie de rupture en fonction de la vitesse de sollicitation d'un système DGEBA/DDM, avec ou sans antiplastifiant [72].

Fracture energy of neat and antiplastified DGEBA/DDM versus rate of deformation [72].

LeMay [50] a étudié l'influence de la longueur du prépolymère pour un système DGEBA/DDS. Il a constaté qu'il existait une relation entre l'énergie de rupture et la masse molaire moyenne entre points de réticulation. $G_{\text {Ic }}$, le taux de restitution d'énergie critique en mode I, est directement proportionnel à $M_{c}^{0,5}$. Cette relation est semblable à celle obtenue dans le cas d'élastomères et d'autres résines époxy au-dessus de leur Tg. LeMay a suggéré que la propagation de fissure dans les époxydes est précédée par un écoulement ductile en extrémité de fissure.

Espuche et al. [61] et [62] ont étudié l'influence de l'ajout d'un extenseur de maille, le méthylcyclohexylamine (MCHA), au système DGEBA/4,4'-diamino-3,3'diméthyldicyclohexylméthane (3 DCM). Cette équipe a montré que les propriétés mécaniques à rupture, mesurées à $25^{\circ} \mathrm{C}$, donc bien au-dessous de $\mathrm{Tg}$, sont reliées au mécanisme de déformation plastique, qui dépend luimême de la température de transition vitreuse des réseaux. La diminution de Tg par ajout de l'extenseur de maille entraîne une diminution de $\sigma_{Y}$ (fig. 17). Cette dernière serait responsable de l'augmentation du facteur d'intensité de contrainte $\mathrm{K}_{\mathrm{Ic}}$ (fig. 18), et du passage d'un régime de propagation instable de fissure à un régime de propagation stable.

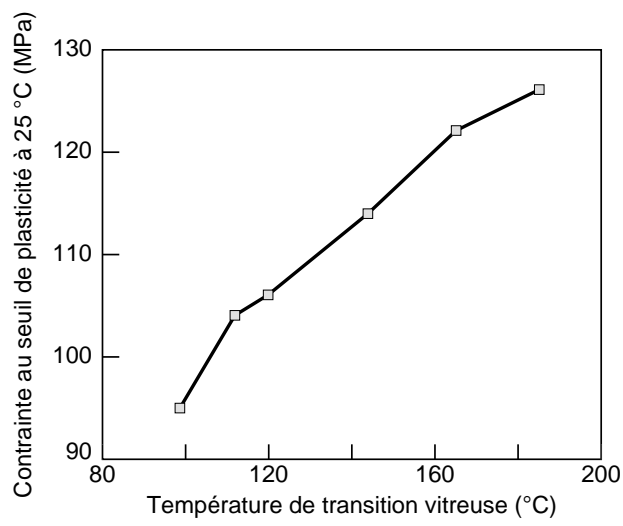

Figure 17

Contrainte au seuil de plasticité en fonction de la température de transition vitreuse pour des réseaux DGEBA/3 DCMMCHA [59].

Yielding stress as a function of glass transition temperature of DGEBA/3 DCM-MCHA networks [59].

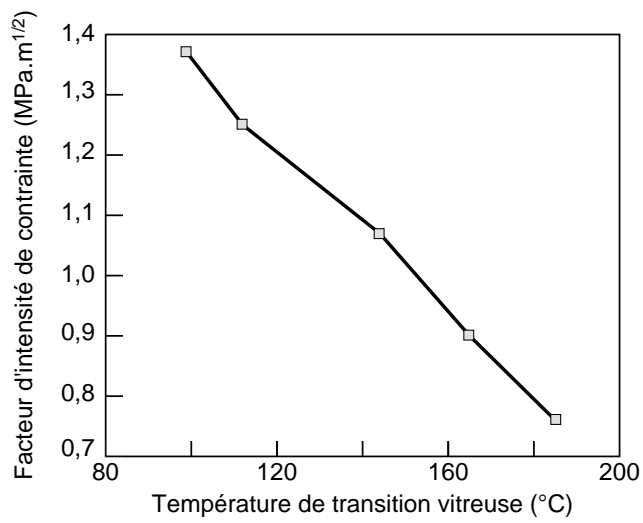

Figure 18

Facteur d'intensité de contrainte en fonction de la température de transition vitreuse pour des réseaux DGEBA/3 DCMMCHA [d'après 59].

Stress intensity factor as a function of glass transition temperature of DGEBA/3 DCM-MCHA networks [after 59].

$\mathrm{Au}$ vu de ces résultats, il est difficile de conclure sur l'influence de tel ou tel paramètre sur le phénomène de rupture. Le manque de données sur la préparation des éprouvettes et les conditions d'essai font que les résultats présentés dans la littérature sont peu comparables. De plus, la technique de modification de la structure des réseaux est primordiale. En effet, la structure chimique (fins de chaînes, fraction soluble, etc.) et la topographie sont différentes selon qu'un extenseur de maille est utilisé ou que les proportions de mélange sont modifiées. 


\section{CONCLUSION}

L'importante étude bibliographique résumée dans cet article permet de faire le point sur l'état actuel des connaissances concernant les résines époxy réticulées par les polyamines, et plus particulièrement sur :

- la réticulation;

- la structure et la morphologie des systèmes réticulés et les paramètres qui les influencent;

- les relations entre la structure et les propriétés.

Il ressort de cette synthèse que beaucoup d'aspects sont encore très discutés, notamment en ce qui concerne les relations entre structure et propriétés dans le domaine des températures d'utilisation, c'est-à-dire entre la transition secondaire $\beta$ et la transition vitreuse. Il est maintenant admis que le module à l'état vitreux est gouverné par l'importance des mouvements des séquences hydroxypropyléther formées lors de la réticulation. En revanche, les résultats concernant les propriétés mécaniques aux grandes déformations, telles que l'allongement et la contrainte à la rupture, ou l'énergie de propagation de fissures, ne permettent pas de conclure. Les tendances obtenues dépendent de la manière utilisée pour modifier la densité de réticulation du système (cuisson, proportions du mélange, extenseurs de mailles, prépolymères de longueur différente), ou encore de l'auteur. Les désaccords entre chercheurs pourraient provenir des conditions de préparation des échantillons, notamment de la vitesse de refroidissement en fin de cuisson qui joue sur les propriétés par l'intermédiaire de contraintes internes résiduelles. De plus, les conditions dans lesquelles sont réalisés les essais mécaniques ne sont pas toujours les mêmes d'un article à l'autre, ce qui rend les comparaisons difficiles.

À la vue de ces conclusions, il semble que des recherches soient encore nécessaires pour essayer de mieux comprendre les relations entre structure et propriétés des résines époxy à l'état vitreux, c'est-à-dire pour les températures d'utilisation de la plupart des applications. Ces résultats pourraient alors être intégrés dans des études visant à mieux comprendre les mécanismes de déchéance des structures composites.

\section{REMERCIEMENTS}

Nous remercions la société Ciba-Geigy, qui nous a fourni les systèmes époxy nécessaires à l'étude menée, ainsi que le professeur J.L. Halary de l'École supérieure de physique et chimie industrielles de la ville de Paris pour son soutien scientifique.

\section{RÉFÉRENCES}

1 May C.A. (1988), Epoxy Resins: Chemistry and Technology, éd. Dekker.

2 Ellis B. (1993), Chemistry and Technology of Epoxy Resins, Blackie Academic \& Professional Ed.

3 Bardonnet P., Résines époxydes : composants et propriétés. In: Techniques de l'ingénieur : traité des plastiques, A3 465.

4 Gulino D., J. Galy, J.P. Pascault, L. Tighzert et Q.T. Pham (1983), Etude des prépolymères époxydes par chromatographie et ${ }^{1} \mathrm{RMN}$ à $350 \mathrm{MHz}$. Die Makromolekulare Chemie, 184 , p. 411-429.

5 Prime R.B. (1981), Thermosets. In: Thermal Characterization of Polymeric Materials. Academic press, p. 435-569.

6 Pascault J.P. (1989), Chimie et mise en œuvre des mélanges réactifs. In: Initiation à la chimie et à la physico-chimie macromoléculaire, 7: matériaux composites à base de polymères, éd. GFP, p. 14-41.

7 St John N.A. et G.A. George (1994), Diglycidyl amine epoxy resin networks: kinetics and mechanisms of cure. Progress in Polymer Science, 19, p. 755-795.

8 Rozenberg B.A. (1986), Kinetics, thermodynamics and mechanisms of reactions of oligomers with amines. Advances in Polymer Science: Epoxy Resins and Composites II, 75, p. 113-165.

9 Wang X. et J.K. Gillham (1991), Competitive primary amine/epoxy and secondary amine/epoxy reactions: effect on the isothermal time-to-vitrify. Journal of Applied Polymer Science, 43, p. 2267-2277.

10 Lovell R. et H. Windle (1990), WAXS investigation of local structure in epoxy networks. Polymer, 31, p. 593-601.

11 Riccardi C.C. et R.J.J. Williams (1986), A kinetic scheme for amine-epoxy reaction with simultaneous etherification. Journal of Applied Polymer Science, 32, p. 3445-3456.

12 Sabra A., T.M. Lam, J.P. Pascault, M.F. Grenier-Loustalot, et P. Grenier (1987), Characterization and behaviour of epoxy-based diaminodiphenylsulfone networks. Polymer, 28, p. $1030-1036$.

13 Cukierman S., R.P. Eustache, J.L. Halary, F. Lauprêtre et L. Monnerie, Processus de déformation des matrices de composite en relation avec leur structure moléculaire. Rapport DRET, $\mathrm{n}^{\circ} 87 / 140$.

14 Eustache R.P. (1990), Étude par RMN ${ }^{13} \mathrm{C}$ haute résolution dans les solides de la structure chimique des résines polyester et de la dynamique moléculaire dans les résines époxy et dans les résines polyester. Thèse de doctorat de l'université Paris VI.

15 Galy J., A. Sabra et J.P. Pascault (1986), Characterization of epoxy thermosetting systems by differential scanning calorimetry. Polymer Engineering and Science, 26, p. 15141523.

16 Gillham J.K. (1986), Curing. In: Encyclopedia of Polymer Science and Engineering, 4, Wiley-Interscience $2^{\text {nde }}$ édition, p. 322-382.

17 Flory P.J. (1971), Principles of Polymer Chemistry, Cornell University Press. 
18 Peng X. et J.K. Gillham (1985), Time-Temperature-Transformation (TTT) cure diagrams: relationship between Tg and the temperature and time of cure for epoxy systems. Journal of Applied Polymer Science, 30, p. 4685-4696.

19 Bardonnet P., Résines époxydes : mise en œuvre et applications. In: Techniques de l'ingénieur : traité des plastiques, A3 466.

20 Plepys A.R. et R.J. Farris (1990), Evolution of residual stresses in three-dimensionally constrained epoxy resins, Polymer, 31, p. 1932-1936.

21 Bauchière D. (1995), Relation entre les caractéristiques physiques de réseaux époxy modèles et le développement de contraintes internes. Thèse de doctorat de l'université Paris VI.

22 Dúsek K. (1986), Network formation in curing of epoxy resin. Advances in Polymer Science: Epoxy Resins and Composites III, 78, p. 1-59.

23 Oleinik E.F. (1986), Epoxy-aromatic amine networks in the glassy state: structure and properties. Advances in Polymer Science: Epoxy Resins and Composites IV, 80, p. 49-99.

24 Gupta V.B., L.T. Drzal, W.W. Adams et R. Omlor (1985), An electron microscopic study of the morphology of cured epoxy resin. Journal of Materials Science, 20, p. 3439-3452.

25 Morgan R.J. (1985), Structure-properties relations of epoxies used as composite matrices. Advances in Polymer Science: Epoxy Resins and Composites I, 72, p. 1-43.

26 Mijovic J. et J.A. Koutsky (1979), Correlation between nodular morphology and fracture properties of cured epoxy resins. Polymer, 20, p. 1095-1107.

27 Wu C.S. (1991), Effect of stirring on morphology and properties in a catalyst cured epoxy system. Journal of Applied Polymer Science, 43, p. 1421-1429.

28 Sanz G., J. Garmendia, M.A. Andres et I. Mondragon (1995), Dependence of dynamic mechanical behavior of DGEBA/ DDM stoichiometric epoxy systems on the conditions of curing process. Journal of Applied Polymer Science, 55, p. 75-87.

29 Cukierman S. (1991), Analyse moléculaire des relations entre structure chimique et propriétés dynamiques mécaniques de réseaux époxy modèles. Thèse de doctorat de l'université Paris VI.

30 Dúsek K., J. Plestil et F. Lednicky (1978), Are cured epoxy resins inhomogeneous? Polymer, 19, p. 393-397.

31 Halary J.L., L. Monnerie, F. Lauprêtre et S. Cukierman (1994), Structure-property relationships in model epoxy networks. Macromolecular Reports, A31 (suppl. 6 et 7), p. 927-934.

32 Gerard J.F., J. Galy, J.P. Pascault, S. Cukierman et J.L. Halary (1991), Viscoelastic response of model epoxy networks in the glass transition region. Polymer Engineering and Science, 31, p. 615-621.

33 Cukierman S., J.L. Halary et L. Monnerie (1991), Dynamic mechanical response of model epoxy networks in the glassy state. Polymer Engineering and Science, 31, p. 1476-1482.

34 Halary J.L., S. Cukierman et L. Monnerie (1989), Relations entre structure chimique et propriétés mécaniques dans les réseaux époxydes. Bulletin de la Société chimique de Belgique, 98, p. 623-634.

35 Danieley N.D. et E.R. Long (1981), Effects of curing on the glass transition temperature and moisture absorption of a neat epoxy resin. Journal of Polymer Science, Polymer Chemistry Edition, 19, p. 2443-2449.

36 Chang T.D., S.H. Carr et J.O. Brittain (1982), Studies of epoxy resin systems. Part B: Effect of crosslinking on the physical properties of an epoxy resin. Polymer Engineering and Science, 22, p. 1213-1220.

37 LeMay J.D. et F.N. Kelley (1986), Structure and ultimate properties of epoxy resins. Advances in Polymer Science: Epoxy Resins and Composites III, 78, p. 115-148.

38 Meyer F., G. Sanz, A. Eceiza, I. Mondragon et J. Mijovik (1995), The effect of stoichiometry and thermal history during cure on structure and properties of epoxy network. Polymer, 36, p. 1407-1414.

39 Min B.G., J.H. Hodgkin et Z.H. Stachurski (1993), The dependence of fracture properties on cure temperature in DGEBA/DDS epoxy system. Journal of Applied Polymer Science, 48, p. 1303-1312.

40 Wisanrakkit G. et J.K. Gillham (1990), The glass transition temperature $(\mathrm{Tg})$ as an index of chemical conversion for a high-Tg amine/epoxy system: chemical and diffusioncontrolled reaction kinetics. Journal of Applied Polymer Science, 41, p. 2885-2929.

41 Murayama T. et J.P. Bell (1970), Relation between the network structure and dynamic mechanical properties of a typical amine-cured epoxy polymer. Journal of Polymer Science, part A-2, 8, p. 437-445.

42 Gupta V.B., L.T. Drzal et C.Y.C Lee (1985), The temperature-dependence of some mechanical properties of cured epoxy resin systems. Polymer Engineering and Science, 25, p. 872-823.

43 Gupta V.B. et L.T. Drzal (1985), The physical basis of moisture transport in a cured epoxy resin system. Journal of Applied Polymer Science, 30, p. 4467-4493.

44 LeMay J.D., B.J. Swetlin et F.N. Kelley (1984), Structure and fracture of highly cross-linked networks. ACS Symposium Series 243, p. 165-183.

45 Kong F.M., C.M. Walkup et R.J. Morgan (1982), The relation between the network structure, deformation and failure processes, and mechanical properties of epoxies. Composites Technology Review, 4, p. 88-93.

46 Morgan R.J., C.M. Walkup, F.M. Kong et E.T. Mones (1985), Development of epoxy matrices for filament-wound graphite structures. 30th National SAMPE Symposium, March 19-21, p. 1209-1220.

47 Vakil U.M. et G.C. Martin (1992), Crosslinked epoxies: network structure characterization and physical-mechanical properties. Journal of Applied Polymer Science, 46, p. 20892099.

48 Misra S.C. J.A. Manson et L.H. Sperling (1979), Effect of cross-link density distribution on the engineering behavior of epoxies. Advances in Chemistry Series, 114, p. 137-156.

49 Banks L. et B. Ellis (1982), The glass transition temperatures of highly crosslinked networks: cured epoxy resins, Polymer, 23, p. 1466-1472.

50 LeMay J.D. (1985), The influence of molecular structure on the mechanical and fracture behavior of highly crosslinked epoxy networks. PhD Thesis of the University of Akron.

51 Bellenger V., E. Morel et J. Verdu (1988), Structureproperties relationships for densely crosslinked epoxideamine systems based on epoxyde or amine mixtures. Part 1: glass transition temperature. Journal of Materials Science, 23, p. 4244-4250.

52 Di Marzio E.A. (1964), On the second-order transition of rubber. Journal of Research of the National Bureau of Standard - A. Physics and Chemistry, 68a, p. 611-617.

53 Fox T.G. et S. Loshaek (1955), Influence of molecular weight and degree of crosslinking on the specific volume and 
glass temperature of polymers. Journal of Polymer Science, 15, p. 371-390.

54 Bellenger V., J. Verdu et E. Morel (1987), Effect of structure on glass transition temperature of amine crosslinked epoxies. Journal of Polymer Science. Part B: Polymer Physics, 25, p. 1219-1234.

55 Dammont F.R. et T.K. Kwei (1967), Dynamic mechanical properties of aromatic, aliphatique, and partially fluorinated epoxy resins. Journal of Polymer Science. Part A-2, 5, p. 761-769.

56 Charlesworth J.M. (1988), Effect of crosslink density on the molecular relaxations in diepoxide-diamine network polymers. Part 1: the glassy region. Polymer Engineering and Science, 28, 4, p. 221-229.

57 Ochi M., M. Okazaki et M. Shimbo (1982), Mechanical relaxation mechanism of epoxide resins cured with aliphatic diamines. Journal of Polymer Science. Polymer Physics Edition, 20, p. 689-699.

58 Monnerie L. (1990), Dynamique locale des polymères et influence sur les propriétés mécaniques. In: Initiation à la chimie et à la physico-chimie macromoléculaires, Vol. 8 : Structure des polymères et méthodes d'études, éd. GFP, p. $183-232$.

59 Daly J., A. Brittenet A. Garton (1984), An additive for increasing the strength and modulus of amine-cured epoxy resins. Journal of Applied Polymer Science, 29, p. 14031414.

60 Caux X., G. Coulon et B. Escaig (1988), Influence of the degree of crosslinking on the plastic deformation behaviour of epoxy resins. Polymer, 29, p. 808-813.

61 Espuche E., J. Galy, J.F. Gérard, J.P. Pascault et H. Sautereau (1995), Influence of crosslinked density and chain flexibility on mechanical properties of model epoxy networks. Macromolecular Symposia 93, p. 107-115.

62 Espuche E., J. Galy, J.F. Gérard, J.P. Pascault et H. Sautereau (1995), Influence de la densité de réticulation et de la flexibilité des chaînes sur les propriétés mécaniques de réseaux époxydes modèles. La Revue de Métallurgie-CIT/ Science et génie des matériaux, p. 1017-1026.

63 Halary J.L., S. Cukierman et L. Monnerie (1992), Influence de la prise d'humidité sur les propriétés dynamiques mécaniques de réseaux époxy modèles. Annales des composites: Composites à matrice polymère et humidité, AMAC, p. 103-112.

64 Enns J.B. et J.K. Gillham ( 1983), Effect of the extent of cure on the modulus, glass transition, water absorption, and density of an amine-cured epoxy. Journal of Applied Polymer Science, 28, p. 2831-2846.

65 Pang K.P. et J.K. Gillham (1989), Anomalous behavior of cured epoxy resins: Density at room temperature versus time and temperature of cure. Journal of Applied Polymer Science, 37, p. 1969-1991.

66 Wang X. et J.K. Gillham (1993), Tg-temperature property (Tg-Tp) diagram for thermosetting systems: anomalous behavior of physical properties vs. extent of cure. Journal of Applied Polymer Science, 47, p. 425-446.

67 Venditti R.A., J.K. Gillham, Y.C. Jean et Y. Lou ( 1995), Free volume after cure vs. fractional conversion for a highTg epoxy/amine thermosetting system. Journal of Applied Polymer Science, 56, p. 1207-1220.

68 Aronhime M.T., X. Peng et J.K. Gillham (1986), Effect of time-temperature path of cure on the water absorption of high Tg epoxy resins. Journal of Applied Polymer Science, 32, p. 3589-3626.

69 Kong E.S.W. (1986), Physical aging in epoxy matrices and composites. Advances in Polymer Science: Epoxy resins and composites IV, 80, p. 125-171.

70 Gupta V.B., L.T. Drazl, C.Y.C. Lee et M.J. Rich (1985), The effect of stoichiometry and structure on the dynamic torsional properties of a cured epoxy resin system. Journal of Macromolecular Science, Physics Edition, B23, p. 435-466.

71 Venditti R.A., J.K. Gillham, Y.C. Jean et Y. Lou (1995), Macroscopic volume, van der waals volume and free volume after cure versus chemical conversion for a high-tg epoxy/amine thermosetting system. Journal of Coatings Technology, 67, 844, p. 47-56.

72 Daly J., A. Britten et A. Garton (1984), An additive for increasing the strength and modulus of amine-cured epoxy resins. Journal of Applied Polymer Science, 29, p. 14031414.

Manuscrit définitif reçu en janvier 1997 